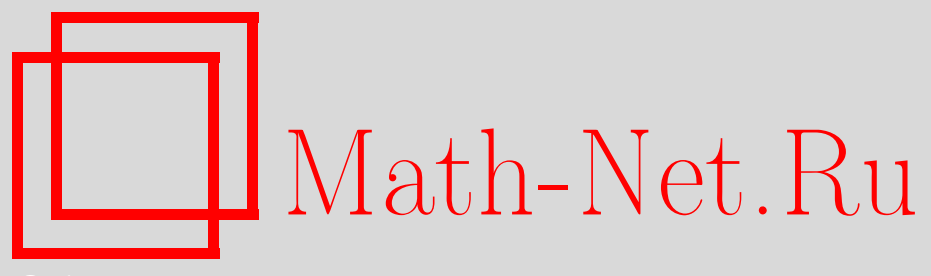

Д. В. Гугнин, Полиномиально зависимые гомоморфизмы. Теорема единственности $n$-гомоморфизмов Фробениуса, УМН, 2007, том 62, выпуск 5, 149-150

DOI: https://doi.org/10.4213/rm8513

Использование Общероссийского математического портала Math-Net.Ru подразумевает, что вы прочитали и согласны с пользовательским соглашением http://www . mathnet.ru/rus/agreement

Параметры загрузки:

IP : 34.229 .45 .116

26 апреля 2023 г., 13:55:05

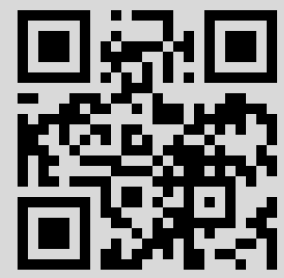




\section{Полиномиально зависимые гомоморфизмы. Теорема единственности $n$-гомоморфизмов Фробениуса}

\section{Д. В. Гугнин}

Понятие $n$-гомоморфизма Фробениуса было введено в 1996-1997 гг. В. М. Бухштабером и Э. Рисом, давшими два различных определения и доказавшими их эквивалентность. Теория развита в [1], [2]. Здесь мы приводим третье определение $n$-гомоморфизма, которое позволяет рассмотреть $n$-гомоморфизмы как частный случай общего понятия полиномиально зависимых гомоморфизмов.

Для симметрического многочлена $p_{n+1}(t)=t_{1}^{n+1}+\cdots+t_{n}^{n+1}$ существует однозначно определенный многочлен $F_{n+1}\left(z_{1}, \ldots, z_{n}\right) \in \mathbb{Q}\left[z_{1}, \ldots, z_{n}\right]$ такой, что $p_{n+1}(t)=$ $F_{n+1}\left(p_{1}(t), \ldots, p_{n}(t)\right)$, где $p_{k}(t)=t_{1}^{k}+\cdots+t_{n}^{k}, 1 \leqslant k \leqslant n,-$ многочлены Ньютона. Многочлен $F_{n+1}\left(z_{1}, \ldots, z_{n}\right)$ мы назовем $n$-м многочленом Фробениуса.

Всюду далее под кольцом $R$ понимается коммутативное кольцо с единицей, содержащее поле рациональных чисел $\mathbb{Q}$, а под алгебрами $A, B, C, \ldots$ - коммутативные ассоциативные $R$-алгебры с единицей.

ОПредЕлЕниЕ 1 . Линейное отображение $R$-алгебр $f: A \rightarrow B$ называется $n$-гомоморфизмом Фробениуса, если выполнены следующие условия:

1) $f\left(1_{A}\right)=n \cdot 1_{B}$

2) $f\left(a^{n+1}\right)=F_{n+1}\left(f a, f a^{2}, \ldots, f a^{n}\right)$ для каждого $a \in A$.

Теорема 1. Пусть $R$ - кольцо, $A-R$-алгебра, $K$-алгебраически замкнутое поле нулевой характеристики и $\sigma: R \rightarrow K$ - гомоморфизм колеи, превращающий $K$ в R-алгебру. Тогда для любого $n$-гомоморфизма $f: A \rightarrow K$ существуют и единственны (с точностъю до перестановок) гомоморфизмы $R$-алгебр $f_{1}, \ldots, f_{n}: A \rightarrow K$ mакие, что $f=f_{1}+\cdots+f_{n}$.

Этот результат при различных дополнительных ограничениях на $R, K$ и $A$ был разными методами получен ранее, сначала В. М. Бухштабером и Э. Рисом в [1], а потом автором в [3]. Доказательство утверждения в общем случае получено автором и использует новый подход, основанный на нетривиальной теореме В. М. Бухштабера и Э. Риса об универсальном $n$-гомоморфизме.

ОПРеДЕЛЕНИЕ 2. Будем называть полиномиальным законом над кольцом $R$ пару $(\mu, \mathscr{P})$, где $\mu \in R$ и $\mathscr{P}$ - некоторое множество многочленов из $\lim R\left[z_{1}, z_{2}, \ldots, z_{k}\right]=$ $R\left[z_{1}, z_{2}, \ldots\right]$, содержащее хотя бы один ненулевой многочлен.

ОПредЕлЕниЕ 3. Линейное отображение $R$-алгебр $f: A \rightarrow B$ называется гомоморфизмом, полиномиально зависимым по закону $(\mu, \mathscr{P})$ (кратко $(\mu, \mathscr{P})$-гомоморфизмом), если

1) $f\left(1_{A}\right)=\mu \cdot 1_{B}$

2) $P\left(f a, f a^{2}, \ldots, f a^{m}\right)=0$ для каждого $a \in A$ и каждого $P\left(z_{1}, z_{2}, \ldots, z_{m}\right) \in \mathscr{P}$.

Ясно, что закон $n$-гомоморфизма Фробениуса является $(\mu, \mathscr{P})$-законом, где $\mu=n$ и $\mathscr{P}=\left\{z_{n+1}-F_{n+1}\left(z_{1}, \ldots, z_{n}\right)\right\}$.

ОПредЕление 4. Два полиномиальных закона $(\mu, \mathscr{P})$ и $(\nu, \mathscr{Q})$ над одним кольцом $R$ называются эквивалентными, если

1) $\mu=\nu$;

2) для любых алгебр $A, B$ множество $(\mu, \mathscr{P})$-гомоморфизмов из $A$ в $B$ совпадает с множеством $(\nu, \mathscr{Q})$-гомоморфизмов из $A$ в $B$.

Данная работа выполнена при финансовой поддержке программы "Ведущие научные школы" (грант НШ 4182.2006.1). 
Имеет место следующая важная лемма.

ЛЕмма 1. 1) Композииия ( $\mu, \mathscr{P})$-гомоморфизма и гомоморфизма алгебр в любом порядке дает снова $(\mu, \mathscr{P})$-гомоморбизм.

2) Для любых алгебр $A, B$ и любого линейного отображения $f: A \rightarrow B$ следующие условия эквивалентны:

a) $f$ является $(\mu, \mathscr{P})$-гомоморфизмом;

b) Композичия $f \circ g_{a}: R[x] \rightarrow B$, где $g_{a}$ - гомоморфизм алгебр $R[x] \rightarrow A, g_{a}(x)=a$, является $(\mu, \mathscr{P})$-гомоморфизмом для каждого $a \in A$.

Эта лемма указывает на особую роль алгебры $R[x]$ при изучении $(\mu, \mathscr{P})$-гомоморфизмов. Более того, можно показать, что существуют так называемая универсальная алгебра $B_{\text {univ }}$, строящаяся по алгебре $R[x]$ и закону $(\mu, \mathscr{P})$, и выделенный $(\mu, \mathscr{P})$-гомоморфизм $f_{\text {univ }}: R[x] \rightarrow B_{\text {univ }}$, называемый универсальным, которые несут полную информацию о законе $(\mu, \mathscr{P})$. С помощью этого $(\mu, \mathscr{P})$-гомоморфизма можно ввести естественное понятие степени закона $(\mu, \mathscr{P})$. Степень - это целое число, большее либо равное -1 . Законы степени -1 и 0 интереса не представляют. Здесь мы дадим другое, более наглядное, определение закона $(\mu, \mathscr{P})$ степени $n \geqslant 1$.

ОПРедЕление 5. Скажем, что $\operatorname{deg}(\mu, \mathscr{P})=n \geqslant 1$, если существуют алгебра $B$ и $(\mu, \mathscr{P})$-гомоморфизм $f: R[x] \rightarrow B$ такие, что элементы $f(x), \ldots, f\left(x^{n}\right)$ алгебраически независимы над $R$ и для любой алгебры $C$ и любого $(\mu, \mathscr{P})$-гомоморфизма $g: R[x] \rightarrow C$ элементы $g(x), \ldots, g\left(x^{n}\right), g\left(x^{n+1}\right)$ алгебраически зависимы над $R$.

ОПределение 6 . Закон $(\mu, \mathscr{P})$ степени $n \geqslant 1$ называется полным, если для любой алгебры $B$ и для любых $b_{1}, \ldots, b_{n} \in B$ существует единственный $(\mu, \mathscr{P})$-гомоморфизм $f: R[x] \rightarrow B$ такой, что $f(x)=b_{1}, f\left(x^{2}\right)=b_{2}, \ldots, f\left(x^{n}\right)=b_{n}$.

Сформулируем главный результат этой статьи - общую теорему единственности.

Теорема 2. 1) Пусть $\mu=n \geqslant 1$. Тогда закон $(\mu, \mathscr{P})$ степени $n$ полон тогда и только тогда, когда он эквивалентен закону п-гомоморфизма Фробениуса.

2) Пусть $(\mu, \mathscr{P})$ - закон степени $n \geqslant 1$. Тогда этот закон является полным тогда и толъко тогда, когда $\mu$-обратимый элемент в $R$ и для любых алгебр $A, B$ и любого линейного отображения $f: A \rightarrow B$ следующие условия эквивалентны:

a) $f$ является $(\mu, \mathscr{P})$-гомоморфизмом;

b) $(n / \mu) f$ является $n$-гомоморфизмом.

Другими словами, закон $(\mu, \mathscr{P})$ получен из закона $n$-гомоморфизма умножением на обратимую константу $(\mu / n)$.

Ключевым и самым сложным этапом доказательства этой теоремы является доказательство так называемой частной теоремы единственности, совпадающей с п. 1) теоремы при условии, что $\mathscr{P}$ состоит только из одного многочлена вида $z_{n+1}-$ $P_{n+1}\left(z_{1}, \ldots, z_{n}\right)$. Доказывается, что многочлен $P_{n+1}\left(z_{1}, \ldots, z_{n}\right)$ должен совпадать с многочленом Фробениуса $F_{n+1}\left(z_{1}, \ldots, z_{n}\right)$. Далее из частной теоремы единственности с помощью универсального ( $\mu, \mathscr{P})$-гомоморфизма выводится п. 1$)$, а из него уже п. 2).

Автор благодарен своему научному руководителю чл.-корр. РАН В. М. Бухштаберу за плодотворные обсуждения и постоянное внимание к работе.

\section{Список литературы}

[1] V. M. Buchstaber, E. G. Rees, Selecta Math. (N.S.), 8:4 (2002), 523-535. [2] B. М. Бухштабер, Е. Г. Рис, УМН, 59:1 (2004), 125-144. [3] Д. В. Гугнин, УМН, 60:5 (2005), 163-164.

Д. В. Гугнин (D. V. Gugnin)

Московский государственный университет им. М. В. Ломоносова

E-mail: dmitry-gugnin@yandex.ru
Представлено В. М. Бухштабером Принято редколлегией 04.08.2007 